\title{
Craniofacial Trauma and Intraocular Surgical Implants
}

\author{
(D) Sunny Chi Lik Au, (1) Simon Tak Chuen Ko \\ Department of Ophthalmology, Tung Wah Eastern Hospital, Hong Kong, China
}

Keywords: Eye, glaucoma, glaucoma drainage implant, eye injuries, eye foreign bodies

\section{Introduction}

Surgical implants are evolving and trending towards a smaller size to facilitate minimally invasive surgery. However, without adequate knowledge, they could be misinterpreted as harmful foreign bodies from trauma.

\section{Case Report}

A 34-year-old male construction site worker suffered from right eye blunt injury while hammering metals, and hit by a metal bar over his right head. He remained conscious without any nausea or dizziness, but attended the emergency department hours later for right eye pain. Applying latanoprost eye drop daily, he volunteered history of right eye uveitic glaucoma with glaucoma implant surgery. The ophthalmologist's record mentioned an ExPRESS (excessive pressure regulating shunt system) shunt (Alcon Laboratories, Fort Worth, Texas, USA) was implanted and was compatible to magnetic resonance imaging (MRI) (1).

Visual acuities were Snellen decimal 0.1 and 0.8 , whereas intraocular pressures (IOP) were 11 and $17 \mathrm{mmHg}$ over right and left eye respectively. Right pupil was irregular with relative afferent pupillary defect. Slit lamp examination found a shiny metal over the superotemporal quadrant of the right eye (Figure 1), plugged by the iris adhering to cornea. There was conjunctival scarring, but no penetrating wounds, subconjunctival haemorrhage or hyphaema. Dilated fundus examination revealed glaucomatous changes without any retinal haemorrhage, commotio retinae, detachment nor rupture. Left eye was unremarkable. Computed tomography scanned for head injury highlighted the hyperdense implant inside the right eye (Figure 2), but no other intraocular foreign bodies nor fracture and intracranial haemorrhage.

\section{Discussion}

Head computed tomography is often scanned for head injury cases, but clinical correlation is essential in case of intraocular surgical implants, particularly their radiological imaging

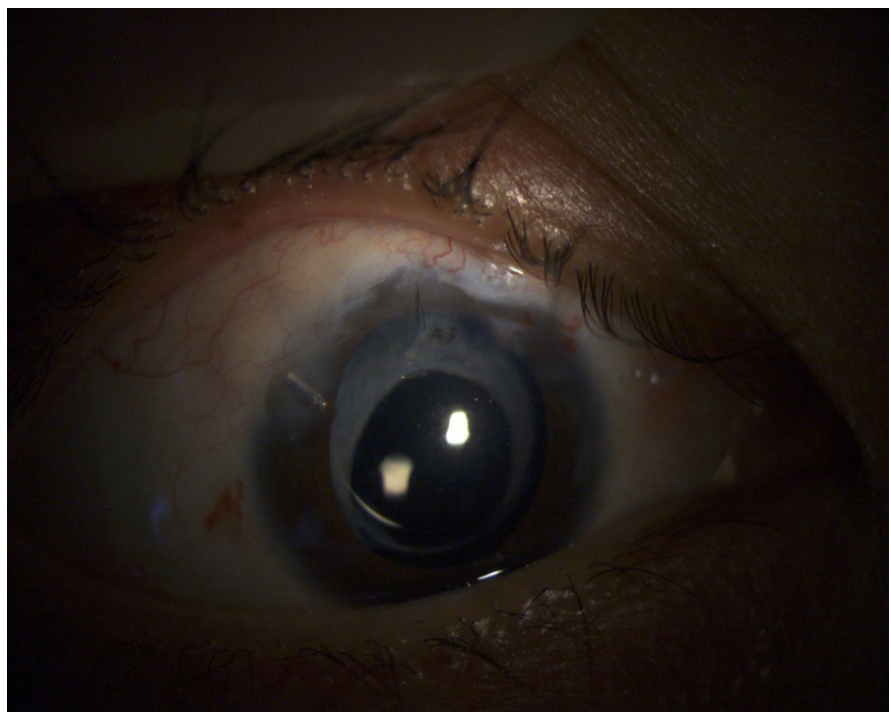

Figure 1. Slit lamp photo of the right eye. The Ex-PRESS shunt, appearing as a tubular metal, was over the superotemporal quadrant of the right eye plugged by the iris adhering to the cornea. No penetrating wound, but scarring over the adjacent conjunctiva suggested previous surgical manipulation. Superior corneal stitch and posterior capsule intraocular lens were also evidenced. 


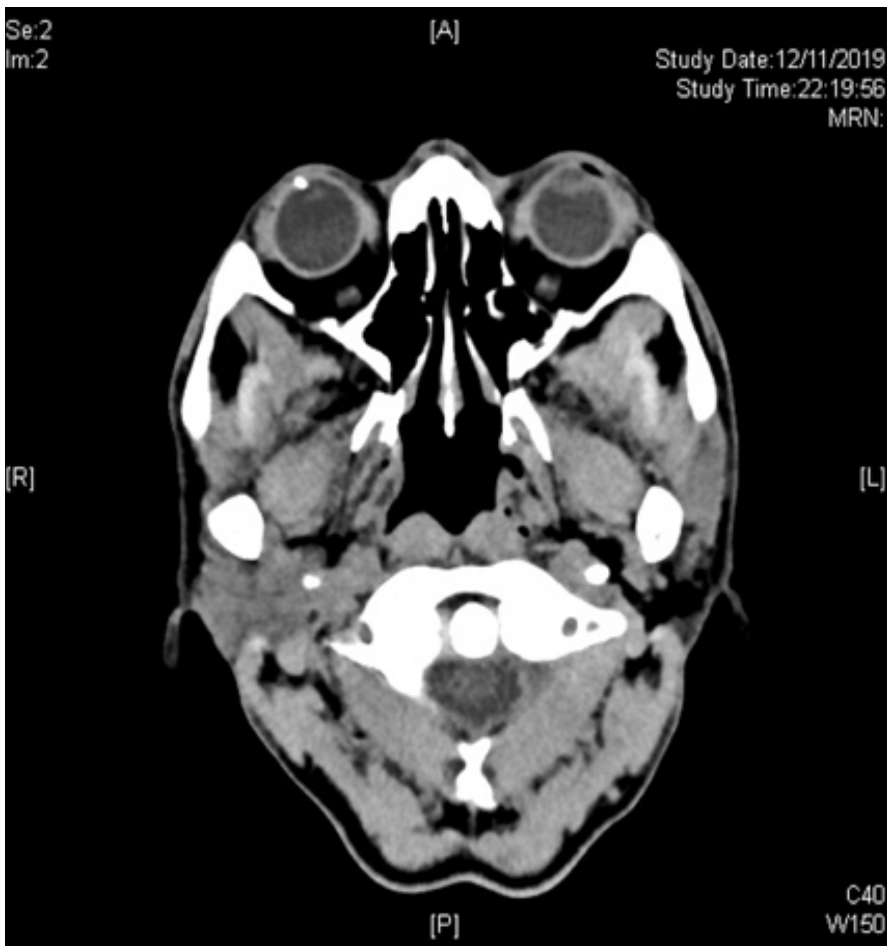

Figure 2. Computed tomography of the head. Transverse cut over the right eye intraocular implant showed its radiologically hyperdense appearance and its location at the anterior chamber in front of the intraocular lens, sticking to the cornea, mimicking a penetrating injury by metallic foreign body

compatibility. Uveitic glaucoma arises from chronic uveitis with persistent elevated IOP (2). In refractory cases that failed pharmacological and traditional trabeculectomy surgery, glaucoma drainage device is the ultimate choice (3). Newer and smaller implants are evolving including the non-valved Baerveldt, valved Ahmed implant and Ex-PRESS. Among them, Ex-PRESS is the smallest $(<3 \mathrm{~mm})$, and the only metallic implant, made of stainless steel, giving its shiny appearance (4). Implanted patient is safe to undergo MRI without disturbing the implant (5).

Normally, we only see the tubular tip end of Ex-PRESS, whereas in our case, it has inferiorly subluxed, but held by the iris sticking it to the cornea. Ophthalmologists were consulted for assessment of the intraocular implant after the trauma, and they offered watch-and-wait conservative treatment with close monitoring of any subsequent rise in IOP. Repair surgery was not indicated unless uncontrollable IOP.

\section{Conclusion}

Intraocular implants are advancing, and smaller in size. This case helps emergency physicians to understand more on differentiating a surgical implant from an exogenous foreign body injury.

\section{Ethics}

Informed Consent: Informed consent was obtained from the patient.

Peer-review: Externally peer-reviewed.

\section{Authorship Contributions}

Surgical and Medical Practices: S.C.L.A., S.T.C.K., Concept: S.C.L.A., Design: S.C.L.A., Data Collection and/or Processing: S.C.L.A., Analysis and/or Interpretation: S.C.L.A., Literature Search: S.C.L.A., Writing: S.C.L.A., S.T.C.K.

Conflict of Interest: No conflict of interest was declared by the authors.

Financial Disclosure: The authors declared that this study received no financial support.

\section{References}

1. Chan JE, Netland PA. EX-PRESS glaucoma filtration device: efficacy, safety, and predictability. Med Devices (Auckl). 2015;8:381-8.

2. Kalogeropoulos D, Sung VC. Pathogenesis of uveitic glaucoma. J Curr Glaucoma Pract. 2018;12:125-38.

3. Lee JW, Chan JCh, Qing L, Lai JS. Early postoperative results and complications of using the EX-PRESS shunt in uncontrolled uveitic glaucoma: a case series of preliminary results. J Curr Glaucoma Pract. 2014;8:20-4.

4. Mermoud A. Ex-PRESS implant. Br J Ophthalmol. 2005;89:396-97.

5. Mabray MC, Uzelac A, Talbott JF, Lin SC, Gean AD. Ex-PRESS glaucoma filter: an MRI compatible metallic orbital foreign body imaged at 1.5 and 3T. Clin Radiol. 2015;70:e28-34. 